\title{
Advanced Radio Resource Allocation Strategies for Satellite Digital Multimedia Broadcast (SDMB) System
}

\author{
Hongfei Du, Victor Y. H. Kueh and Barry G. Evans \\ Center for Communication Systems Research, Univeristy of Surrey, Guildford, Surrey, GU2 7XH, UK \\ H.Du@surrey.ac.uk
}

Abstract-This paper presents a novel approach for the radio resource allocation as part of the overall Radio Resource Management scheme for the Satellite Digital Multimedia Broadcast (SDMB) system. A two-stage bin-packing algorithm is designed and employed for channel mapping which considers multiplexing at both the transport and physical channels so as to achieve the highest possible degree of utilization and efficiency of the available system capacity. Evaluations of these schemes are performed for various traffic mixes and physical channel configuration scenarios. packing

Keywords-SDMB, MBMS, radio resource allocation, bin-

\section{INTRODUCTION}

The Satellite Digital Multimedia Broadcast (SDMB) system implements a satellite based broadcast layer over $2.5 \mathrm{G}$ and $3 \mathrm{G}$ terrestrial mobile cellular networks aiming at the efficient delivery of the Multimedia Broadcast Multicast Service (MBMS). Due to the unidirectional nature of the SDMB system and the point-to-multipoint services it provides, the Radio Resource Management (RRM) functionalities implemented at the SDMB access layer, which comprise packet scheduling, radio resource allocation (RRA), admission control and interactions with higher transport layer protocols, are challenging. This paper considers the design and evaluation of suitable and efficient RRA schemes for SDMB, as part of the work carried out within the framework of the EU IST MAESTRO project [1] and the joint activity on the RRM for satellite-UMTS within the SatNEx project [2].

The Radio Resource Allocation (RRA) is responsible for the Radio Bearer (RB) configuration, which includes the estimation of the required number of logical/transport/physical channels and their mapping together with the actual Transport Format Combination Set (TFCS) for each physical channel. Previous research on the channel mapping has used a conventional single-stage bin-packing algorithm [3], which assumes that the MBMS point-to-multipoint Traffic CHannel (MTCH) logical channels are mapped one-to-one onto the Forward Access Channel (FACH) transport channels, which are subsequently multiplexed onto the Secondary Common Control Physical CHannel (S-CCPCH). This type of channel mapping which considers only a single-level of multiplexing at the physical layer is shown in Fig 1-a. The problem with this simple one-to-one mapping at the transport channel is that there exists residual capacity on the transport channel which is not utilized when the bit rate of the logical channel does not exactly match the corresponding bit rate of the transport channel, i.e. the $\mathrm{MTCH}$ rate is less than the $\mathrm{FACH}$ rate.

In order to resolve this inefficiency in resource utilization, a two-level multiplexing structure at both the transport channel and the physical channel is considered in the channel mapping strategy of the RRA, as shown in Fig 1-b. At the first level of multiplexing, multiple MTCHs are mapped onto a single FACH (logical channel multiplexing), whereas the mapping of several FACHs onto the S-CCPCH (transport channel multiplexing) is regarded as the second level of multiplexing. Although having logical channel multiplexing is more complex compared to the one-to-one mapping strategy in terms of processing complexity, it benefits from minimum residual capacity on the $\mathrm{FACH}$ as the $\mathrm{FACH}$ can be assigned with more MTCHs as long as they can be accommodated. With regard to the two-level bin-packing algorithm, whose objective is to pack the given items into the minimum possible number of bins [4], in this paper, the MTCHs will be referred to as items to be packed; the S-CCPCHs as bins; while the FACHs will be denoted as intermediate-bins. The objective of the overall mapping is to achieve the highest possible degree of utilization and efficiency of available system capacity whilst meeting the QoS requirements.

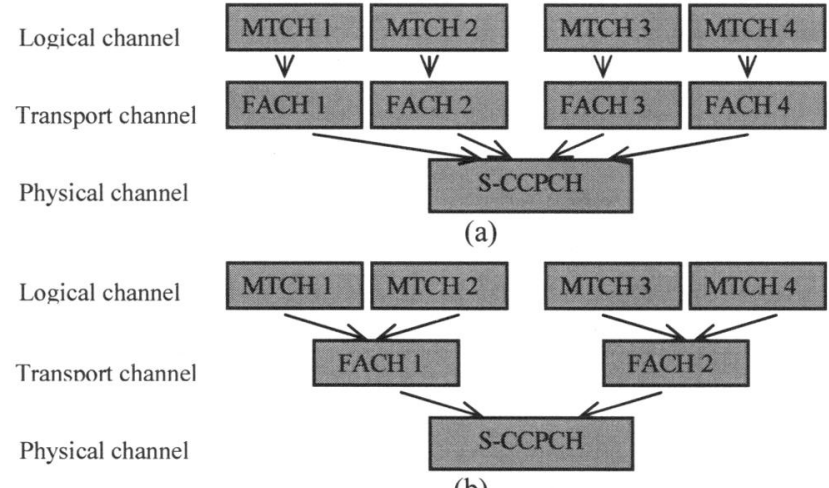

(b)

Figure 1. Channel mapping options considering (a) Transport channel multiplexing (b) Logical and transport channel multiplexing

\section{RAdio Resource Allocation Algorithm}

Previous research on single-stage bin-packing algorithms split the RRA task into three main steps [3]. In the context of the proposed two-stage bin-packing algorithm, these are extended into four main steps as described in the following:

\section{Step 1: Estimation of required MTCHS}

Let $\mathrm{S}$ be the set of different streaming services. A streaming service can be characterized by the 3-tuple - arrival rate $\lambda_{i}$, duration $\mu_{i}$ and requested rate for each type of service $R_{i}$ [5]. The cardinality of the service set is $N$, i.e. $|S|=N$. No assumption is made for the flow burstiness; the flow might be of constant bit rate (CBR) or variable bit rate (VBR), but in the latter case the $R_{i}$. value is set to the mean/guaranteed 
rate attribute. Each element $S_{i}$ corresponds to a member of the service set, i.e. a streaming service.

Let $P_{b l}$ be a vector of size $N$ corresponding to blocking probabilities targeted for each streaming service, i.e. there is one-to-one correspondence between $S_{i}$ and $P_{b l}^{i}$.

Then the required MTCHs for each $S_{i}$ can be derived via use of well-known results of classical queuing theory [4]:

- From the m-server loss queuing system, for each service type $S_{i}$ separately, i.e. invocation of the $\mathrm{M} / \mathrm{M} / \mathrm{m} / \mathrm{m}$ formula $\mathrm{N}$ times.

- From the extension of the Erlang formula to the multiple services scenario over all types of flows $S_{i}$ requesting the same rate $R_{i}$, irrespective of the arrival rates or service durations of the individual services.

In both cases, the required number of MTCHs is the number of servers that will guarantee the target blocking probabilities $P_{b l}^{i}$.

\section{Step 2: Estimation of required FACHs}

This is the additional step in the extension of the singlestage mapping to the two-stage mapping algorithm, whereby the objective is to estimate the number of intermediate-bins (FACHs) required, which will be used in the next step of the two-stage channel mapping. There are two proposed approaches to estimate and determine the required number of FACHs in the two-level channel multiplexing.

The first approach is to specify the intermediate-bin a priori according to the available bit rate of $\mathrm{FACH}$ using the following rules:

- The total bit rate of the FACHs should not be less than the total bit rate of the MTCHs.

- A wide range of bit rate of the FACHs should be made available to choose from so as to provide the maximum possible degree of selectivity and satisfaction for the different rates of the MTCHs. This is to allow the different mapping schemes to be employed in the next step.

The MTCHs are then mapped onto the predefined FACHs using different bin-packing algorithm, as will be described in step 3. The inter-relationship and inter-dependence between two stages can be illustrated in Fig.2:

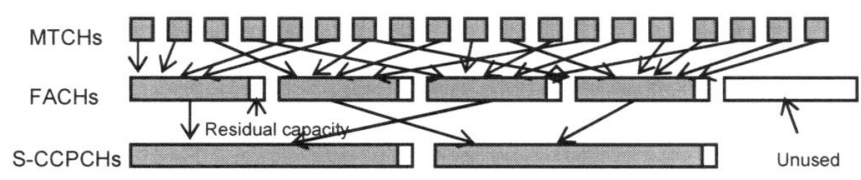

Figure 2. Inter-relationship between the two multiplexing stages

As shown in Fig.2, after the first stage of multiplexing, only the utilized FACHs are mapped onto the S-CCPCHs, whilst the unused FACHs are not mapped onto the S-CCPCHs, which will lead to better utilization of both $\mathrm{FACHs} /$ CCPCHs.

An alternative approach to estimate the required number of FACHs is based on the bit rates required by the MTCHs and the available bit rates of the FACHs, with the aim to minimise both the number of required FACHs as well as the residual capacity on the FACHs. A brief description of this algorithm is as follows.

Let $R$ be a vector of size of size $\mathrm{N}$, corresponding to the rate for each $M T C H$, i.e. $\left\{R_{1}, R_{2}, R_{3}, \quad, R_{N}\right\}$. The available rate of FACHs is $S=\left\{\mathrm{S}_{1}, \mathrm{~S}_{2}, \mathrm{~S}_{3}, \ldots\right\}$. The required number of FACHs will be set according to the vectors $R$ and $S$. The elements in both $R$ and $S$ are first reordered in decreasing order of bit rates, and vector $\mathrm{D}=[\mathrm{]}$ is predefined as the output of FACHs. Then the bit rates of MTCHs are selected from $R$ one by one and compared to the available FACHs' rate $S$. If a set of $R_{i}$ can be fully mapped into a single $S_{j}$, then the rate $S_{j}$ is assigned as one of the required FACHs; otherwise the next lower rate of $\mathrm{FACH} \mathrm{S}_{\mathrm{j}+1}$ is chosen and checked if it can accommodate the MTCH. The loop continues until all the MTCHs are assigned. A detailed description of this algorithm and the evaluation of this approach can be found in [8].

\section{Step 3: Mapping of the MTCHs - FACHs - S-CCPCHs}

The objective of this step is to achieve the two-level mapping of the derived MTCHs onto FACHs, and then mapping of the derived FACHs onto the available S-CCPCHs using bin-packing algorithm, given that the number of available FACHs is $\mathrm{M}$, and their capacity is $\mathrm{c}_{\mathrm{j}}$. The number of available S-CCPCHs is $\mathrm{K}$ and their maximum capacity $\mathrm{c}$, are known a priori.

The conventional single-stage bin-packing approach, which does not take into account the power requirement $(\mathrm{Eb} / \mathrm{No})$ of individual services, is extended herein and developed for the two-stage bin-packing scenario. The mathematical formulation that gives the two-stage bin-packing solution could be [6]:

$$
\begin{aligned}
\text { minimize: } & \mathrm{Z}=\sum_{\mathrm{k}=1}^{K} z_{\mathrm{k}} ; \mathrm{Y}=\sum_{\mathrm{j}=1}^{M} \mathrm{y}_{j} ; \mathrm{w}=\sum_{j=1}^{M} r_{j} \\
\text { subject to: } & \sum_{\mathrm{i}=1}^{N} R_{i} x_{i j}=c_{j} y_{j}+r_{j} ; \mathrm{j} \in\{1 \ldots M\} ; \\
& \sum_{j=1}^{M} \mathrm{c}_{\mathrm{j}} y_{j} \leq c z_{k} \\
& \sum_{\mathrm{j}=1}^{M} x_{i j}=1 ; i \in\{1 \ldots N\} ; \sum_{\mathrm{k}=1}^{K} y_{j k}=1 ; \mathrm{j} \in\{1 \ldots M\}
\end{aligned}
$$

where $z_{\mathrm{k}}=1$ if bin $\mathrm{k}$ is used, 0 otherwise; $y_{j}=1$ if intermediate-bin $\mathrm{j}$ is used, 0 otherwise; and $x_{i j}=1$, if item $\mathrm{i}$ is assigned to intermediate-bin $\mathrm{j}, 0$ otherwise; and $y_{j k}=1$, if intermediate-bin $\mathrm{j}$ is assigned to bin $\mathrm{k}, 0$ otherwise. $r_{\mathrm{j}}$ is the residual capacity of each intermediate-bin $\mathrm{j}$. The condition for a feasible solution of the problem should be: $\{\mathrm{Z} \leq \mathrm{M} \leq \mathrm{N}\}$.

\section{Step 4: Derivation of TCFS for each S-CCPCH}

The TCFS should be broad enough to capture the packetlevel dynamics of the expected services over some future time interval. The chosen transport block size is in line with the 
acket sizes coming from the applications in order to .ninimize the overheads (headers and padding). A generic approach for deriving this TFCS can be found in [5].

The method described above is only applicable for streaming services. On the other hand, for the dimensioning of background services, which cannot be characterized in terms of $\left\{\lambda_{i}, \mu_{i}, R_{i}\right\}$, the residual capacity corresponding to FACHs can be estimated from the residual rates per S-CCPCH, i.e. the FACHs are spread over the available S-CCPCHs and each S$\mathrm{CCPCH}$ is filled with one $\mathrm{FACH}$ of the residual rate. Therefore, the network makes available the remaining capacities in $\mathrm{FACHs} / \mathrm{S}-\mathrm{CCPCHs}$ for those services. Negotiations on the accepted rate for those services may be in accordance with the service priority level for optimum utilization.

\section{EVALUATION OF RRA SCHEMES}

The types of user service supported within the SDMB system are streaming and download, which corresponds to UMTS QoS class (streaming, background) [5]. Streaming is a sequence of "moving images" and sound that are delivered in ompressed form and played directly at the user terminal, whereby the media is sent in a continuous stream and is played as it arrives. For the download services, the multimedia contents are pre-stored in the local cache for later processing, whereby the file cannot be played until it is downloaded completely. Within the context of SDMB system, the download services can be further classified, according to its sensitivity to delay, into two types: "hot download" and "cold download". Hot download is defined to consist of content, which is delay-sensitive, e.g. broadcasting of urgent messages and monitoring of variable data. Cold download, on the other hand, consists of content, which is inherently not sensitive to change during the delay of the transmission.

The two-stage bin-packing algorithm has been evaluated for various traffic mixes and physical channel configuration scenarios, as highlighted below:

- Traffic mix of $80 \%$ streaming - $20 \%$ download; $50 \%$ streaming $-50 \%$ download; $20 \%$ streaming $-80 \%$ download;

- $\quad$ S-CCPCH configurations of $3 \times 384 \mathrm{kbps} ; 2 \times 384$ kbps; 1 × 384 kbps; 3 x 128kbps; 1 × 384kbps +3 x128 kbps

The traffic mixes herein refer to the capacity allocated to (reserved for) each type of services (streaming, download) assuming implicitly a fixed boundary for the capacity. Note that in some cases, the partitioning of capacity between streaming and download services shown in the scenarios do not follow the division of $x \%$ streaming-(100-x) $\%$ download traffic mix exactly due to the implicit use of 'fixed' bearers of $32 \mathrm{kbps}, 64 \mathrm{kbps}, 128 \mathrm{kbps}$ and $256 \mathrm{kbps}$ for the streaming class.

Due to the space limitation, only the evaluation results of the channel mapping for $3 \mathrm{~S}-\mathrm{CCPCHs}$ of $384 \mathrm{kbps}$ each with traffic mix of $80 \%$ streaming - $20 \%$ download, and for $3 \mathrm{~S}$ $\mathrm{CCPCHs}$ of $128 \mathrm{kbps}$ each with traffic mix of $50 \%$ streaming - 50\% download are shown in this paper. For both scenarios, a different bin-packing algorithm is used at each stage of mapping, whereby the streaming services carried by the MTCHs are first mapped onto the FACHs via the FF binpacking algorithm. These FACHs are then mapped onto the SCCPCHs via the BF bin-packing algorithm, and the download services are then assigned to the residual capacity of S$\mathrm{CCPCHs}$ according to the specified percentage of the traffic mix for download. Another approach for the download service allocation is utilizing the residual capacity of FACHs for the download services after mapping the streaming MTCHs onto the available FACHs, in that case it is assumed that there exists residual capacity on the FACHs after the first stage channel mapping.

As for the bin-packing algorithms, the following wellknown approaches are used to implement the packing process [6]:

- Next-Fit (NF) bin-packing algorithm: each item is assigned to the current bin if it fits; otherwise, it is assigned to the next new bin, which becomes the current bin.

- First-Fit (FF) bin-packing algorithm: the items are considered according to increasing indices and each item is assigned to the lowest indexed initialized bin into which it fits. Only when the current item cannot fit into any initialized bin, a new bin is introduced.

- Best-Fit (BF) bin-packing algorithm: the current item is assigned to a feasible bin (if any) having the smallest residual capacity.

There are actually different choices in choosing the binpacking algorithm for channel mapping at the two stages, either:

- Utilizing the same bin-packing algorithm at each stage, e.g. using NF for both MTCH->FACH and FACH->S$\mathrm{CCPCH}$; or

- Utilizing a combination of bin-packing algorithms at each stage, e.g. FF for $\mathrm{MTCH}->\mathrm{FACH}$ and $\mathrm{BF}$ for FACH->S-CCPCH;

Table I shows the system level parameters $\left\{\lambda_{i}, \mu_{i}, R_{i}\right\}$ characterising the streaming SDMB user services for the case of $80 \%$ streaming - $20 \%$ download given $3 \mathrm{~S}-\mathrm{CCPCHs}$ of 384 kbps each, while Table II depicts the values when the traffic mix is $50 \%$ streaming - $50 \%$ download given $3 \mathrm{~S}-\mathrm{CCPCHs}$ of $128 \mathrm{kbps}$ each. Note that the load is defined to be the product of the 3-tuple - bit rate, system arrival rate and the service duration:

Load $=\frac{\text { system-level arrival rate } \times \text { mean service duration } \times \text { guaranted bit rate }}{3600}$ while the normalized load is defined to be the load of each application as a percentage of the total channel capacity:

$$
\text { Normalized load/application }=\frac{\{\text { Load(kbps) for each application }\}}{\{\text { Total physical channel capacity }\}}
$$

The required number of streaming MTCHs and FACHs varies for a given service group/characterization and target QoS requirements. Table III and V show the required number of streaming MTCHs and FACHs for both scenarios stated 
above, respectively. QoS requirement is implemented by means of limiting the maximum allowable blocking probability in the algorithm.

TABLE I. STREAMING SERVICE CHARACTERIZATION FOR 80\% STREAMING -20\% DOWNLOAD GIVEN $3 \times 384 \mathrm{KBPS}$ S-CCPCH

\begin{tabular}{|c|c|c|c|c|c|c|}
\hline $\begin{array}{l}\text { Application } \\
\text { type }\end{array}$ & $\begin{array}{l}\text { Guaranteed } \\
\text { bit rate } \\
\text { (kbps) }\end{array}$ & $\begin{array}{c}\text { Mean } \\
\text { service } \\
\text { duration } \\
\text { (sec) }\end{array}$ & $\begin{array}{l}\text { System- } \\
\text { level } \\
\text { arrival } \\
\text { rate } \\
\text { (per } \\
\text { hour) }\end{array}$ & $\begin{array}{l}\text { Load } \\
\text { (kbps) }\end{array}$ & $\begin{array}{l}\text { Normalized } \\
\text { load per } \\
\text { application } \\
\text { (percent) }\end{array}$ & $\begin{array}{l}\text { Normalized } \\
\text { load per } \\
\text { application } \\
\text { type } \\
\text { (percent) }\end{array}$ \\
\hline \multirow{2}{*}{$\begin{array}{c}\text { Audio } \\
\text { broadcast }\end{array}$} & 32 & \multirow{2}{*}{300} & 2.75 & 7.333 & 0.637 & \multirow{2}{*}{2.225} \\
\hline & 64 & & 3.43 & 18.29 & 1.588 & \\
\hline \multirow{3}{*}{$\begin{array}{c}\text { Video } \\
\text { broadcast }\end{array}$} & 64 & \multirow{3}{*}{300} & 1.67 & 8.907 & 0.773 & \multirow{3}{*}{2.940} \\
\hline & 128 & & 0.12 & 1.28 & 0.111 & \\
\hline & 256 & & 1.11 & 23.68 & 2.056 & \\
\hline
\end{tabular}

TABLE II. STREAMING SERVICE CHARACTERIZATION FOR 50\% STREAMING $-50 \%$ DOWNLOAD GIVEN $3 \times 128$ KBPS S-CCPCH

\begin{tabular}{|c|c|c|c|c|c|}
\hline $\begin{array}{c}\text { Application } \\
\text { type }\end{array}$ & $\begin{array}{c}\text { Guaranteed } \\
\text { bit rate } \\
\text { (kbps) }\end{array}$ & $\begin{array}{c}\text { Mean } \\
\text { service } \\
\text { duration } \\
\text { (sec) }\end{array}$ & $\begin{array}{c}\text { System-level } \\
\text { arrival rate } \\
\text { (per hour) }\end{array}$ & $\begin{array}{c}\text { Load } \\
\text { (kbps) }\end{array}$ & $\begin{array}{c}\text { Normalized } \\
\text { load per } \\
\text { application } \\
\text { (percent) }\end{array}$ \\
\hline $\begin{array}{c}\text { Audio } \\
\text { broadcast }\end{array}$ & 32 & 300 & 1.8 & 4.8 & 1.25 \\
\hline $\begin{array}{c}\text { Audio } \\
\text { broadcast }\end{array}$ & 128 & 300 & 0.12 & 1.28 & 0.33 \\
\hline
\end{tabular}

A1: Channel mapping configuration for the scenario of $3 \mathrm{~S}$ CCPCH of $384 \mathrm{kbps}$ each with traffic mix of $80 \%$ streaming $-20 \%$ download

TABLE III. REQUIRED STREAMING MTCHS AND FACHS FOR 80\% STREAMING-20\% DOWNLOAD GIVEN $3 \times 384$ KBPS S-CCPCH

\begin{tabular}{|c|c|c|c|c|}
\hline MTCH rate & $\mathbf{3 2 k b p s}$ & 64kbps & 128kbps & 256kbps \\
\hline \# of streaming MTCHs & 3 & 3 & 1 & 2 \\
\hline FACH rate & $\mathbf{3 2 k b p s}$ & $\mathbf{6 4 k b p s}$ & $\mathbf{1 2 8 k b p s}$ & $\mathbf{2 5 6 \mathrm { kbps }}$ \\
\hline \# of streaming FACHs & - & - & 2 & 3 \\
\hline
\end{tabular}

TABLE IV. MAPPING DERIVED FOR $80 \%$ STREAMING - $20 \%$ DOWNLOAD GIVEN $3 \times 384$ KBPS S-CCPCH

\begin{tabular}{|c|c|c|c|c|c|c|}
\hline S-CCPCH & \multicolumn{2}{|c|}{1} & \multicolumn{2}{|c|}{2} & \multicolumn{2}{|c|}{3} \\
\hline Bit rate(kbps) & \multicolumn{2}{|c|}{384} & \multicolumn{2}{|c|}{384} & \multicolumn{2}{|c|}{384} \\
\hline $\begin{array}{c}\text { Streaming FACHs } \\
(\mathrm{kbps})\end{array}$ & \multicolumn{2}{|c|}{$\begin{array}{l}256 \times 1 \\
128 \times 1 \\
\end{array}$} & \multicolumn{2}{|c|}{$\begin{array}{l}256 \times 1 \\
128 \times 1 \\
\end{array}$} & \multicolumn{2}{|c|}{$256 \times 1$} \\
\hline $\begin{array}{c}\text { Streaming FACHs } \\
\text { sum( kbps) }\end{array}$ & \multicolumn{2}{|c|}{384} & \multicolumn{2}{|c|}{384} & \multicolumn{2}{|c|}{256} \\
\hline $\begin{array}{c}\text { Download FACHs } \\
\text { (kbps) }\end{array}$ & \multicolumn{2}{|c|}{ - } & \multicolumn{2}{|c|}{-} & \multicolumn{2}{|c|}{$128 \times 1$} \\
\hline $\begin{array}{c}\text { Download FACHs } \\
\text { sum (kbps) }\end{array}$ & - & - & \multicolumn{2}{|c|}{ - } & \multicolumn{2}{|c|}{128} \\
\hline FACH & 1 & 2 & 3 & 4 & 5 & 6 \\
\hline Bit rate(kbps) & 256 & 128 & 256 & 128 & 256 & 128 \\
\hline $\begin{array}{c}\text { Streaming MTCHs } \\
\text { (kbps) }\end{array}$ & $256 \times 1$ & $\begin{array}{l}64 \times 1 \\
32 \times 2 \\
\end{array}$ & $256 \times 1$ & $32 \times 1$ & $\begin{array}{l}128 \times 1 \\
64 \times 2\end{array}$ & - \\
\hline $\begin{array}{c}\text { Streaming MTCHs } \\
\text { sum (kbps) }\end{array}$ & 256 & 128 & 256 & 32 & 256 & - \\
\hline $\begin{array}{c}\text { Download MTCHs } \\
\text { (kbps) }\end{array}$ & - & - & - & - & - & $64 \times 2$ \\
\hline $\begin{array}{c}\text { Download MTCHs } \\
\text { sum (kbps) }\end{array}$ & - & - & - & - & - & 128 \\
\hline
\end{tabular}

As can be seen in Table IV, after the mapping for th streaming services has been performed, there exist two types of residual capacity - the first is the residual capacity of 96 kbps on FACH 4, which cannot be utilized further and hence appears as a waste of FACH capacity; the other is the residual capacity on S-CCPCH 3, which can be assigned to download FACHs for carrying download services. For instance, the 128 kbps residual capacity on $\mathrm{S}-\mathrm{CCPCH} 3$ is allocated to one download $\mathrm{FACH}$, which in turn is assigned equally in capacity to 2 MTCHs of $64 \mathrm{kbps}$ each, so as to accommodate two download applications. The overall channel mapping configuration for this scenario is clearly illustrated in Fig. 3, which also depicts both the residual capacity on the FACH (which goes unutilized) and on the S-CCPCH (which is utilized to carry download services).

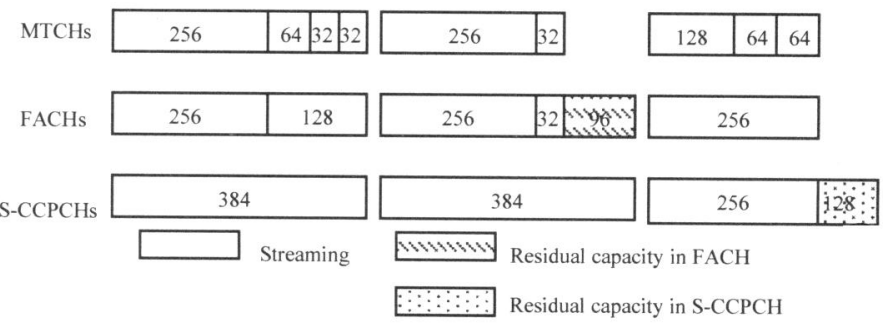

Figure 3. Channel mapping structure utilizing $\mathrm{FF}+\mathrm{BF}$ bin-packing algorithm combination for $80 \%$ streaming-20\% download traffic mix

Note that in some scenarios, priority may be needed for the download service allocation. It has been mentioned earlier that within the context of the SDMB system, for the download services, there exist "cold download" and "hot download" with different sensitivity to delay. In this case, the "hot download" service will be given a higher-priority treatment due to its stringent delay requirement, and thus a higher-rate MTCH will be allocated to the higher priority download service, e.g. given a download FACH of $128 \mathrm{kbps}$ as above, this capacity may be divided accordingly to the priority of the download MTCHs - for instance, "hot download" service can be assigned to a MTCH of $96 \mathrm{kbps}$, while "cold download" service is assigned to a MTCH of $32 \mathrm{kbps}$. The objective of this priority-based download service allocation is to perform better utilization of transport/physical channels for download services with different QoS requirements.

A2: Channel mapping configuration for the scenario of $3 \mathrm{~S}$ CCPCH of $128 \mathrm{kbps}$ each with traffic mix of $50 \%$ streaming $50 \%$ download

TABLE V. REQUIRED STREAMING MTCHS AND FACHS FOR 50\% STREAMING - 50\% DOWNLOAD GIVEN $3 \times 128$ KBPS S-CCPCH

\begin{tabular}{|c|c|c|c|c|}
\hline MTCH rate & $32 \mathrm{kbps}$ & $64 \mathrm{kbps}$ & $\mathbf{1 2 8 \mathrm { kbps }}$ & $\mathbf{2 5 6 \mathrm { kbps }}$ \\
\hline \# of streaming MTCHs & 2 & - & 1 & - \\
\hline FACH rate & $\mathbf{3 2 k b p s}$ & $64 \mathrm{kbps}$ & $128 \mathrm{kbps}$ & $\mathbf{2 5 6 \mathrm { kbps }}$ \\
\hline \# of streaming FACHs & - & 1 & 1 & - \\
\hline
\end{tabular}

Table VI shows a simpler channel mapping configuration compared to Table IV, whereby the same total rate is allocated to the download and streaming services, given a traffic mix of $50 \%-50 \%$. There is one S-CCPCH of rate $128 \mathrm{kbps}$ each for streaming and download service, while the other S-CCPCH of 
$28 \mathrm{kbps}$ is assigned to carry $64 \mathrm{kbps}$ of streaming $\mathrm{FACH}$ and $4 \mathrm{kbps}$ of download FACH.

TABLE VI. MAPPING DERIVED FOR 50\% STREAMING - 50\% DOWNLOAD GIVEN $3 \times 128$ KBPS S-CCPCH

\begin{tabular}{|c|c|c|c|c|}
\hline S-CCPCH & $\mathbf{1}$ & \multicolumn{2}{|c|}{$\mathbf{2}$} & $\mathbf{3}$ \\
\hline Bit rate(kbps) & 128 & \multicolumn{2}{|c|}{128} & 128 \\
\hline Streaming FACHs(kbps) & $128 \times 1$ & \multicolumn{2}{|c|}{$64 \times 1$} & - \\
\hline Streaming FACHs sum (kbps) & 128 & \multicolumn{2}{|c|}{64} & - \\
\hline Download FACHs (kbps) & - & \multicolumn{2}{|c|}{$64 \times 1$} & $128 \times 1$ \\
\hline Download FACHs sum (kbps) & - & \multicolumn{2}{|c|}{64} & 128 \\
\hline FACH & $\mathbf{1}$ & $\mathbf{2}$ & $\mathbf{3}$ & 4 \\
\hline Bit rate(kbps) & 128 & 64 & 64 & 128 \\
\hline Streaming MTCHs(kbps) & $128 \times 1$ & $32 \times 2$ & - & - \\
\hline Streaming MTCHs sum (kbps) & 128 & 64 & - & - \\
\hline Download MTCHs (kbps) & - & - & $32 \times 2$ & $64 \times 2$ \\
\hline Download MTCHs sum (kbps) & - & - & 64 & 128 \\
\hline
\end{tabular}

B: Comparison of transport channels required between single level and two-level multiplexing

Table VII shows the comparison of the required number of ACHs (for both streaming and download) between the ingle-stage and two-stage mapping algorithm, where it clearly shows the advantage of having the two-level multiplexing in that the required number of FACHs is greatly reduced. The reason is that in the two-level multiplexing, multiple MTCHs can be mapped onto a single FACH thereby improving the utilization of FACHs, as compared to the oneto-one mapping of $\mathrm{MTCH}$ to $\mathrm{FACHs}$ in the single-level multiplexing scenario. By having fewer FACHs, this in effect means that less FACHs will be multiplexed onto a single S$\mathrm{CCPCH}$, and therefore the size of the transport format combination set (TFCS) is reduced.

TABLE VII. COMPARISON OF NUMBER OF FACHS REQUIRED BETWEEN SINGLE-STAGE AND TWO-STAGE BIN-PACKING FOR $80 \%$ STREAMING $-20 \%$ DOWNLOAD GIVEN $3 \times 384$ KBPS S-CCPCH

\begin{tabular}{|c|c|c|c|c|}
\hline Single level & $\mathbf{3 2 k b p s}$ & $\mathbf{6 4 k b p s}$ & $\mathbf{1 2 8 k b p s}$ & $\mathbf{2 5 6 \mathbf { k b p s }}$ \\
\hline \# of MTCHs & 3 & 5 & 1 & 2 \\
\hline \# of FACHs & 3 & 5 & 1 & 2 \\
\hline 2 level & $\mathbf{3 2 k b p s}$ & $\mathbf{6 4 k b p s}$ & $\mathbf{1 2 8 \mathrm { kbps }}$ & $\mathbf{2 5 6 \mathrm { kbps }}$ \\
\hline \# of MTCHs & 3 & 5 & 1 & 2 \\
\hline \# of FACHs & - & - & 3 & 3 \\
\hline
\end{tabular}

C: Comparison of mapping results for different combination of bin-packing algorithms applied at each multiplexing level

Table VIII shows the comparison of mapping results for different combinations of bin-packing algorithms (NF+NF; $\mathrm{BF}+\mathrm{BF} ; \mathrm{FF}+\mathrm{BF})$ implemented at the two levels of multiplexing. As mentioned in section II, the FACHs are predefined as a vector according to their available bit rate. The MTCHs can then be mapped onto those predefined FACHs using the different bin-packing algorithm. From the results shown in Table VIII, it can be seen that although the BF algorithm gives the least residual capacity, it requires the largest number of streaming FACHs. This is because the FACHs are predefined as a vector with randomicity as long as this satisfies the different requested $\mathrm{MTCH}$ bit rates, and the corresponding $\mathrm{FACH}$ that is selected is of similar bit rate to the MTCH's provided that the MTCH could be accommodated and a minimum residual capacity is obtained.
On the other hand, the FF algorithm leads to fewer streaming FACHs channels, but the trade-off is that there exists some residual capacity at the transport channel. When NF is employed at both stages, it requires as many as $4 \mathrm{~S}-\mathrm{CCPCH}$ and the cumulative residual capacity on the $\mathrm{FACH} / \mathrm{S}-\mathrm{CCPCH}$ is the largest, which demonstrates the inefficiency of the NF algorithm.

TABLE VIII. COMPARISON OF RESULTS FOR DIFFERENT BIN-PACKING ALGORITHM COMBINATION EMPLOYED AT THE TWO-STAGE FOR 80\% STREAMING - 20\% DOWNLOAD GIVEN $3 \times 384$ KBPS S-CCPCH

\begin{tabular}{|c|c|c|c|c|}
\hline $\begin{array}{c}\text { Bin-packing } \\
\text { algorithm } \\
\text { combination }\end{array}$ & $\begin{array}{c}\text { \# of } \\
\text { streaming } \\
\text { FACHs }\end{array}$ & $\begin{array}{c}\text { Residual capacity } \\
\text { on streaming } \\
\text { FACHs }\end{array}$ & $\begin{array}{c}\text { \# of S- } \\
\text { CCPCHs }\end{array}$ & $\begin{array}{c}\text { Residual } \\
\text { capacity on S- } \\
\text { CCPCHs }\end{array}$ \\
\hline NF+NF & 5 & $96 \mathrm{kbps}$ & 4 & $512 \mathrm{kbps}$ \\
\hline BF+BF & 9 & 0 & 3 & $224 \mathrm{kbps}$ \\
\hline FF+BF & 5 & $96 \mathrm{kbps}$ & 3 & $128 \mathrm{kbps}$ \\
\hline
\end{tabular}

\section{CONCLUSIONS}

Efficient management of the radio resources is one of the main objectives of the SDMB system. Within the RRM strategies, the RRA plays an important role in achieving the maximum utilization and efficiency of the available system capacity. In this paper, a two-stage bin-packing algorithm is developed and employed when channel mapping with two levels of multiplexing is considered. Evaluations comparing these advanced algorithms with the single-stage mapping approach are carried out for various scenarios comprising different traffic mixes, service characterization, and physical channel configurations. These results show the beneficial impact on the utilization of the system capacity and the overall network performance of the SDMB system.

\section{ACKNOWLEDGMENTS}

This work has been supported by the "SatNEx" NoE project(contract No. 507052) - joint activity 2430 (QOS\&RRM) and the IST MAESTRO project (IST-2003507023) under the 6th framework of the European Commission

\section{REFERENCES}

[1] http://ist-maestro.dyndns.org/MAESTRO

[2] http://www.satnex.org/

[3] EU IST SATIN Deliverable 7, "Simulation Results and Evaluation", March 2003

[4] D. Gross, C. M. Harris, "Fundamentals of queuing theory", 2PndP edition, John Wiley and Sons, 1985

[5] EU MAESTRO Deliverable D3-2v1, "SDMB Access Layer Evaluation", December 2004.

[6] S. Martelo, P. Toth, "Knapsack problems: Algorithms and computer implementations", John Wiley \& Sons Ltd., 1990.

[7] M.Karaliopoulos, P.Henrio, K.Narenthiran, E.Angelou, B.G.Evans, "Packet scheduling for the delivery of multicast and broadcast services over S-UMTS", John Wiley \& Sons Ltd. 2004

[8] H.Du, V. Y. H. Kueh and B.G.Evans, "Optimization of Radio Resource Allocation Algorithms for 2-level Channel Multiplexing in SDMB System", July 2005, submitted. 